\title{
Elastic Problem for a Ring of Uniform Force in an Infinite Body ${ }^{1}$
}

\author{
William H. Pell
}

\begin{abstract}
Kelvin has given an integral representation for the displacements in an infinite elastic body produced by a body force acting in an arbitrary portion of this body. By a limit procedure, solutions have been found for point and line singularities acting in the interior of such a body. A similar method is used in this paper to obtain the displacements produced by a uniform force applied along, and normal to, a circle lying in the interior of an infinite elastic body.
\end{abstract}

\section{Introduction}

One of the important results of the classicial theory of elasticity is the solution of Kelvin $[1,3]$ for the displacements in an infinite elastic solid due to a body force distribution throughout all, or a part of the body. From this, one obtains by a limiting procedure the displacements for the case in which the body force reduces to a concentrated force at some point in the infinite solid. This solution is singular at the point of application of the concentrated force, and plays a role for the Navier equations of elasticity analogous to that which the potential of a point charge plays for Laplace's equation. By the process of superposition, the displacements, due to any finite number of concentrated forces with different directions and at different points, can be obtained. The principle of superposition and a limiting process such as is used in mechanics to obtain a concentrated moment can be employed to obtain solutions for higher-order singularities. For example, one may superpose the solutions for point forces $P$, a distance $h$ apart, and with opposite senses. By allowing $h \rightarrow 0$ while $P h$ remains constant, the solution corresponding to a "double force" is obtained. Superposition of three of these with mutually perpendicular lines of action gives the "center of compression". In this way, a catalogue of singular solutions can be built up. A number of such solutions has been given by Boussinesq [4] and Dougall [5], and, more recently, using other methods, Mindlin [6] has given an extensive list of such "nuclei of strain", as they have been called by Love.

By superposition of these nuclei of strain in our infinite solid, one may hope to be able to solve problems for domains with boundaries, and this has been done in a number of important cases, e. g., the half space under concentrated normal load on its boundary, or concentrated load on a line, or over an area such as a circle or rectangle [7].

The Kelvin integrals will be used here to obtain the displacements due to a concentrated force applied along, and normal to a circle in an infinite solid. While no mathematical features of particular novelty arise in the analysis, the solution does exhibit an interesting use of elliptic integrals. The problem has been undertaken as a first step in the application of such methods to problems dealing with bodies having axial symmetry; in particular, it is hoped to use the result obtained here as a first step in obtaining the solution due to a ring of pressure applied to the surface of an elastic cone. It is therefore natural to carry out the work in spherical, rather than the somewhat simpler cylindrical, coordinates, and this has been done.

\section{Kelvin's Integral of the Navier Equations}

Consider an elastic solid of infinite extent, and let its material points be referred to rectangular cartesian coordinates $x_{i}, i=1,2,3$. If a body force with components $F_{j}\left(x_{1}, x_{2}, x_{3}\right)$,

1 This research was supported by the United States Air Force, through the Office of Scientific Research of the Air Research and Development Command. 
$j=1,2,3$, per unit volume acts within a region $V$ of the solid, and $u_{j}\left(x_{1}, x_{2}, x_{3}\right), j=1,2,3$ represent the displacement components in the solid, then the Navier equations in $V$ are

$$
\mu \nabla^{2} u_{i}+(\lambda+\mu) \frac{\partial}{\partial x_{i}} \Delta=-F_{i}, \quad i=1,2,3,
$$

where $\lambda$ and $\mu$ are Lame's elastic constants, and $\Delta$ is the sum of the extensional strains. The right side of (2.1) vanishes outside of $V$.

Lord Kelvin [1,3] has given a solution of these equations, valid throughout the solid, which represents the effects of the body force, viz.,

$$
u_{i}(x)=A \int_{V}\left[B \frac{F_{i}(\xi)}{R}-\frac{\partial}{\partial x_{i}}\left(\frac{1}{R}\right) \sum_{j=1}^{3}\left(x_{j}-\xi_{j}\right) F_{j}(\xi)\right] d V, \quad i=1,2,3
$$

where $\xi_{j}, j=1,2,3$ are variables of integration, $x$ and $\xi$ have been used to indicate the three variables $x_{j}$ and $\xi_{j}$, and

$$
\begin{gathered}
R^{2}=\sum_{j=1}^{3}\left(x_{j}-\xi_{j}\right)^{2}, \\
A=\frac{\lambda+\mu}{8 \mu \pi(\lambda+2 \mu)}, \quad B=\frac{\lambda+3 \mu}{\lambda+\mu} .
\end{gathered}
$$

\section{The Kelvin Integrals in Spherical Coordinates}

It will be convenient for our purposes to obtain the spherical coordinate equivalents of (2.2), i. e., to obtain displacement components in the spherical coordinate directions expressed in terms of spherical coordinates.

Let $r, \theta$, and $\phi$ be spherical coordinates related to the $x_{i}$ coordinates by

$$
\begin{aligned}
& x_{1}=r \sin \theta \cos \phi, \\
& x_{2}=r \sin \theta \sin \phi, \\
& x_{3}=r \cos \theta,
\end{aligned}
$$

as indicated in figure 1 . If $V_{i}(x)$ denote the components of a vector in the $x_{i}$ coordinate system

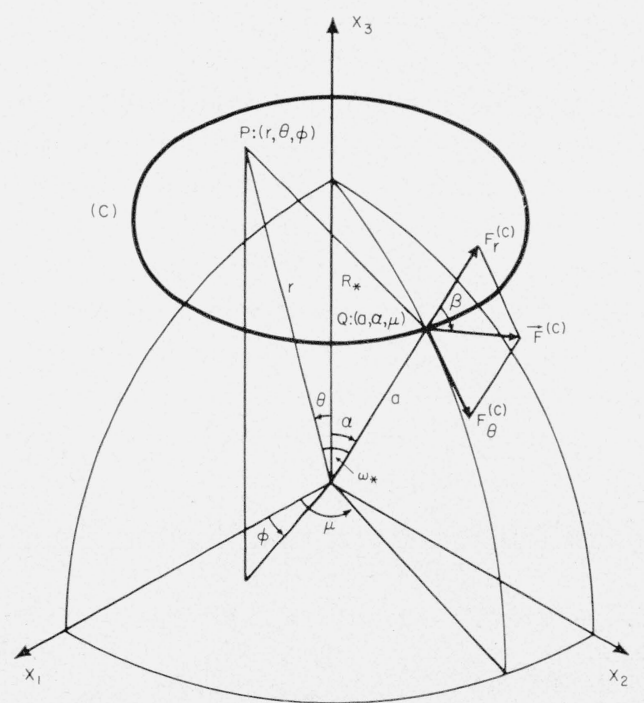

Figure 1. Concentrated force applied to a ring. 
and $V_{r}(r, \theta, \phi), V_{\theta}(r, \theta, \phi), V_{\phi}(r, \theta, \phi)$ its components in spherical coordinates, then it is easily shown either by tensor methods or by elementary geometry that

$$
\begin{aligned}
& V_{r}=\left(V_{1} \cos \phi+V_{2} \sin \phi\right) \sin \theta+V_{3} \cos \theta, \\
& V_{\theta}=\left(V_{1} \cos \phi+V_{2} \sin \phi\right) \cos \theta-V_{3} \sin \theta, \\
& V_{\phi}=-V_{1} \sin \phi+V_{2} \cos \phi .
\end{aligned}
$$

Inversion of this system gives

$$
\begin{aligned}
& V_{1}=\left(V_{r} \sin \theta+V_{\theta} \cos \theta\right) \cos \phi-V_{\phi} \sin \phi, \\
& V_{2}=\left(V_{r} \sin \theta+V_{\theta} \cos \theta\right) \sin \phi+V_{\phi} \cos \phi, \\
& V_{3}=V_{r} \cos \theta-V_{\theta} \sin \theta .
\end{aligned}
$$

If we let $u_{r}, u_{\theta}, u_{\phi}$ be the components of the elastic displacement in spherical coordinates, then they are connected with the cartesian components $u_{i}$ through (3.2) with $V_{r}$, etc., replaced by $u_{r}$, etc., and $V_{i}$ replaced by $u_{i}$. If we suppose this done, and the expressions (2.2) for $u_{i}$ inserted in the result, then we obtain, after some simplification,

$$
\begin{aligned}
& v_{r}(r, \theta, \phi)=A \int_{V}\left\{B \frac{\overline{F_{r}}}{R}+\frac{1}{R^{3}}\left[r \overline{F_{r}}-\rho F_{r}\right][r-\rho \cos \omega]\right\} d V, \\
& v_{\theta}(r, \theta, \phi)=A \int_{V}\left\{B \frac{\overline{F_{\theta}}}{R}+\frac{\rho}{R^{3}}\left[r \overline{F_{r}}-\rho F_{r}\right][\sin \theta \cos \lambda-\cos \theta \sin \lambda \cos (\phi-\mu)]\right\} d V, \\
& v_{\phi}(r, \theta, \phi)=A \int_{V}\left\{B \frac{\overline{F_{\phi}}}{R}+\frac{\rho}{R^{3}}\left[r \overline{F_{r}}-\rho F_{r}\right] \sin \lambda \sin (\phi-\mu)\right\} d V,
\end{aligned}
$$

where $\rho, \lambda, \mu$ are the values of $r, \theta, \phi$, respectively, associated with the integration variables $\xi_{i}$. It is easy to verify that

$$
\begin{aligned}
& R^{2}=r^{2}+\rho^{2}-2 r \rho \cos \omega, \\
& \cos \omega=\cos \theta \cos \lambda+\sin \theta \sin \lambda \cos (\phi-\mu),
\end{aligned}
$$

i. e., $R$ is the distance between the fixed point $(r, \theta, \phi)$ at which the displacements are to be calculated and the variable point of integration $(\rho, \lambda, \mu)$, while $\omega$ is the angle between the radii vectors $r$ and $\rho$. We let $F_{r}$, etc., be the components of the body force in spherical coordinates. The arguments of $F_{r}$ in (3.4) are $\rho, \lambda, \mu$, but $\overline{F_{r}}$ etc., are used to designate certain terms which are as yet incompletely expressed in spherical coordinates, viz,

$$
\begin{aligned}
& \overline{F_{r}}=\left[F_{1}(\xi) \cos \phi+F_{2}(\xi) \sin \phi\right] \sin \theta+F_{3}(\xi) \cos \theta, \\
& \overline{F_{\theta}}=\left[F_{1}(\xi) \cos \phi+F_{2}(\xi) \sin \phi\right] \cos \theta-F_{3}(\xi) \sin \theta, \\
& \bar{F}_{\phi}=-F_{1}(\xi) \sin \phi+F_{2}(\xi) \cos \phi .
\end{aligned}
$$

But the $F_{i}(\xi)$ are given in terms of $F_{r}(\rho, \lambda, \mu)$, etc., by (3.3) with the obvious replacements, and hence we find that

$$
\begin{aligned}
& \bar{F}_{r}=F_{r} \cos \omega-F_{\theta} \Omega+F_{\phi} \sin \theta \sin (\phi-\mu), \\
& \bar{F}_{\theta}=-F_{r} \Lambda+F_{\theta} \Xi+F_{\phi} \cos \theta \sin (\phi-\mu), \\
& \bar{F}_{\phi}=-\left(F_{r} \sin \lambda+F_{\theta} \cos \lambda\right) \sin (\phi-\mu)+F_{\phi} \cos (\phi-\mu),
\end{aligned}
$$


where the arguments of $F_{r}$, etc., are $\rho, \lambda, \mu$, and

$$
\begin{aligned}
& \Xi=\sin \theta \sin \lambda+\cos \theta \cos \lambda \cos (\mu-\phi), \\
& \Lambda=\sin \theta \cos \lambda-\cos \theta \sin \lambda \cos (\mu-\phi), \\
& \Omega=\cos \theta \sin \lambda-\sin \theta \cos \lambda \cos (\mu-\phi) .
\end{aligned}
$$

With the insertion of (3.7) in (3.4), we obtain the displacements in spherical coordinates obtained from the Kelvin integrals:

$$
\begin{gathered}
v_{r}=A \int_{V}\left\{\frac { B } { R } \left[\left(F_{r} \cos \omega-F_{\theta} \Omega+F_{\phi} \sin \theta \sin (\phi-\mu)\right]\right.\right. \\
\left.\quad+\frac{r-\rho \cos \omega}{R^{3}}\left[F_{r}(r \cos \omega-\rho)-F_{\theta} r \Omega+r F_{\phi} \sin \theta \sin (\phi-\mu)\right]\right\} d V, \\
v_{\theta}=A \int_{V}\left\{\frac{B}{R}\left[-\Lambda F_{r}+\Xi F_{\theta}+F_{\phi} \cos ^{-} \theta \sin (\phi-\mu)\right]+\frac{\rho \Lambda}{R^{3}}\left[F_{r}(r \cos \omega-\rho)\right.\right. \\
\left.\left.\quad-F_{\theta} r \Omega+r F_{\phi} \sin \theta \sin (\phi-\mu)\right]\right\} d V, \\
v_{\phi}=A \int_{V}\left\{\frac{B}{R}\left[-\left(F_{r} \sin \lambda+F_{\theta} \cos \lambda\right) \sin (\phi-\mu)+F_{\phi} \cos (\phi-\mu)\right]\right. \\
\quad-\frac{\rho}{R^{3}} \sin \lambda \sin (\phi-\mu)\left[F_{r}(r \cos \omega-\rho)-F_{\theta} r \Omega+r F_{\phi} \sin \theta \sin (\phi-\mu)\right\} d V .
\end{gathered}
$$

\section{The Displacement Integrals for a Ring of Concentrated Force}

The concept of a concentrated force $\vec{F}^{(c)}$ acting at a point $P$ of an infinite body may be arrived at in the following way. We first define a distributed body force which vanishes outside some volume $\Delta V$ containing $P$ in its interior, and inside $\Delta V$ is some continuous $\vec{F}$ which has the property that

$$
\vec{F}^{(c)}=\int_{\Delta V} \vec{F} d V
$$

Now $\Delta V$ is let tend to zero, always with $P$ in its interior, and $\vec{F}$ allowed to increase in such a way that the value of the integral maintains the constant value $\vec{F}^{(c)}$. Thus

$$
\vec{F}^{(c)}(P)=\lim _{\Delta V \rightarrow 0} \int_{\Delta V} \vec{F} d V
$$

We wish to consider a continuous distribution of concentrated force along a circle $(C)$ whose center lies on the $x_{3}$ axis, and whose plane is parallel to that of the $x_{1}$ and $x_{2}$ axes. (See fig. 1.) In spherical coordinates we take the equation of the circle to be

$$
r=a, \theta=\alpha,
$$

where $0<a<\infty, 0<\alpha<\pi$. Suppose $\vec{F}(\phi)=\left(F_{r}(\phi), F_{\theta}(\phi), 0\right)$ is a continuous distribution of force defined in the region $a-\Delta r<r<a+\Delta r, \alpha-\Delta \theta<\theta<\alpha+\Delta \theta \mu-\Delta \phi<\phi<\mu+\Delta \phi$ enclosing the point $P:(a, \alpha, \mu)$. For simplicity, let us consider only the component $F_{\theta}$. In general, because the $\theta$-direction varies from point to point in $\Delta V$, this distribution of force will have a nonzero resultant force and moment with respect to the coordinate directions at $(a, \alpha, \mu)$. It is easy to show, however, that if the resultant $F_{\theta}{ }^{(c)}$ in the $\theta$-direction is held fixed as we let $\Delta r, \Delta \theta, \Delta \phi$ tend 
to zero, then we obtain in the limit only a force $F_{\theta}{ }^{(c)}$ acting in the $\theta$-direction. The same remark holds for the component $F_{r}(\phi)$. In this way we obtain a concentrated force $\vec{F}^{(c)}(\mu)=\left(F_{r}{ }_{r}^{(c)}:(\mu)\right.$, $\left.F_{\theta}{ }^{(c)}(\mu), 0\right)$, acting at $\phi=\mu$ on the circle $r=a, \theta=\alpha$. This force lies in the plane $\phi=\mu$, and is therefore normal to the ring.

The displacements corresponding to the distribution $\vec{F}=\left(F_{r}, F_{\theta}, 0\right)$ in $\Delta V$ are seen from (3.9) to be

$$
\begin{aligned}
& v_{r}=A \int_{\Delta V}\left\{\frac{B}{R}\left[F_{r} \cos \omega-F_{\theta} \Omega\right]+\frac{1}{R^{3}}[r-\rho \cos \omega]\left[F_{r}(r \cos \omega-\rho)-r \Omega F_{\theta}\right]\right\} d V, \\
& \left.v_{\theta}=A \int_{\Delta V}\left\{\frac{B}{R}\left[-\Lambda F_{r}+\Xi F_{\theta}\right]+\frac{\rho \Lambda}{R^{3}} F_{r}(r \cos \omega-\rho)-r \Omega F_{\theta}\right]\right\} d V, \\
& v_{\phi}=A \int_{\Delta V}\left\{-\frac{B}{R}\left[F_{r} \sin \lambda+F_{\theta} \cos \lambda\right]+\frac{\rho}{R^{3}} \sin \lambda\left[F_{r}^{\prime}(r \cos \omega-\rho)-r \Omega F_{\theta}\right]\right\} \sin (\phi-\mu) d V,
\end{aligned}
$$

where $d V=\rho^{2} \sin \lambda d \rho d \lambda d \mu$. Each of these expressions consists of a sum of terms of the form

$$
\int_{\Delta} V^{f(r, \theta, \phi ; \rho, \lambda, \mu) F(\phi) d V}
$$

where $F(\phi)$ is either $F_{r}(\phi)$ or $F_{\theta}(\phi)$, and $f$ represents its coefficient. It is not difficult to show that if $(r, \theta, \phi) \neq(a, \alpha, \mu)$, then under the assumed behavior of $\vec{F}$ in (4.1),

$$
\lim _{\Delta V \rightarrow 0} \int_{\Delta V} f(r, \theta, \phi ; \rho, \lambda, \mu) F(\phi) d V=f(r, \theta, \phi ; a, \alpha, \mu) F^{(c)}(\mu) .
$$

Accordingly, if we now consider a distribution of force of strength $\vec{F}^{(c)}$ per unit of arc length along the circle $(C)$, we obtain for the displacements at $(r, \theta, \phi)$ the expressions

$$
\begin{aligned}
v_{r}= & A \int_{0}^{2 \pi} \frac{1}{R_{*}}\left\{B\left[F_{r}{ }^{(c)}(\mu) \cos \omega_{*}-\Omega_{*} F_{\theta}{ }^{(c)}(\mu)\right]+\right. \\
& \left.\frac{1}{R_{*}{ }^{2}}\left[r-a \cos \omega_{*}\right]\left[F_{r}{ }^{(c)}(\mu)\left(r \cos \omega_{*}-a\right)-r \Omega_{*} F_{\theta}{ }^{(c)}(\mu)\right]\right\} d \mu, \\
v_{\theta}= & A \int_{0}^{2 \pi} \frac{1}{R_{*}}\left\{B\left[-F_{r}{ }^{(c)}(\mu) \Lambda_{*}+F_{\theta}{ }^{(c)}(\mu) \Xi_{*}\right]+\right. \\
& \left.\frac{a \Lambda_{*}}{R_{*}{ }^{2}}\left[F_{r}{ }^{(c)}(\mu)\left(r \cos \omega_{*}-a\right)-r \Omega_{*} F_{\theta}{ }^{(c)}(\mu)\right]\right\} d \mu, \\
v_{\phi}= & A \int_{0}^{2 \pi} \frac{1}{R_{*}}\left\{-B\left[F_{r}{ }^{(c)}(\mu) \sin \alpha+F_{\theta}{ }^{(c)}(\mu) \cos \alpha\right]+\right. \\
& \left.\frac{a \sin \alpha}{R_{*}{ }^{2}}\left[F_{r}{ }^{(c)}(\mu)\left(r \cos \omega_{*}-a\right)-r \Omega_{*} F_{\theta}{ }^{(c)}(\mu)\right]\right\} \sin (\phi-\mu) d \mu,
\end{aligned}
$$

where $*$ indicates evaluation of the quantity to which it is attached at $\rho=a, \lambda=\alpha$, i. e.,

$$
\begin{aligned}
R_{*}{ }^{2} & =r^{2}+a^{2}-2 a r \cos \omega_{*}, \\
\cos \omega_{*} & =\cos \theta \cos \alpha+\sin \theta \sin \alpha \cos (\mu-\phi), \\
\Xi_{*} & =\sin \theta \sin \alpha+\cos \theta \cos \alpha \cos (\mu-\phi), \\
\Lambda_{*} & =\sin \theta \cos \alpha-\cos \theta \sin \alpha \cos (\mu-\phi), \text { and } \\
\Omega_{*} & =\cos \theta \sin \alpha-\sin \theta \cos \alpha \cos (\mu-\phi) .
\end{aligned}
$$

The evaluation of the integrals occurring in (4.4) will be difficult, or impossible, in finite 
terms, unless $F_{r}$ and $F_{\theta}$ have the form of trigonometric polynomials. More general functions $F_{r}$ and $F_{\theta}$ may be replaced by their Fourier series expansions. This leads to an infinite series for each displacement component, the terms of which contain integrals evaluable in terms of complete elliptic integrals of the first and second kinds. The process of such evaluation involves the use of recursion formulas which lead to extremely long and cumbersome expressions. We shall therefore restrict our consideration to the case in which the components $F_{r}{ }^{(c)}$ and $F_{\theta}{ }^{(c}$ are constant. This does not mean that the direction of the force is the same at every point of the ring, but that at every point it lies in the plane of the point and the $x_{3}$ axis, and has the same orientation in this plane. (See fig. 1.) If we denote the magnitude of this force by $P$ and its angle with the $r$-direction by $\beta$, where the positive sense of $\beta$ is the same as that of $\theta$, then $F_{r}=P \cos \beta$ and ${ }^{*} F_{\theta}=P \sin \beta$, and (4.4) may be written as

$$
\begin{aligned}
& v_{r}=A P \int_{0}^{2 \pi} \frac{1}{R_{*}}\left\{B g_{1}+\frac{1}{R_{*}^{2}}\left(r-a \cos \omega_{*}\right)\left(r g_{1}-a \cos \omega_{*}\right)\right\} d \mu, \\
& v_{\theta}=A P \int_{0}^{2 \pi} \frac{1}{R_{*}}\left\{B g_{2}+\frac{a}{R_{*}^{2}} \Lambda_{*}\left(r g_{1}-a \cos \omega_{*}\right)\right\} d \mu,
\end{aligned}
$$

where

$$
\begin{gathered}
g_{1}(\theta, \phi, \alpha, \beta, \mu)=\cos (\alpha+\beta) \cos \theta+\sin (\alpha+\beta) \sin \theta \cos (\mu-\phi), \\
g_{2}(\theta, \phi, \alpha, \beta, \mu)=-\cos (\alpha+\beta) \sin \theta+\sin (\alpha+\beta) \cos \theta \cos (\mu-\phi) .
\end{gathered}
$$

The third integral of (4.4) vanishes, since the integrand is an odd function with respect to $\mu=\pi$. It is now convenient to introduce nondimensional quantities $\eta$ and $h$ defined by

$$
r=\eta a, \quad R_{*}=a h .
$$

Thus

and (4.6) become

$$
h^{2}=1+\eta^{2}-2 \eta \cos \omega_{*},
$$

$$
\begin{aligned}
& v_{r}=\frac{A P}{a} \int_{0}^{2 \pi} \frac{1}{h}\left\{B g_{1}+\frac{1}{h^{2}}\left(\eta-\cos \omega_{*}\right)\left(\eta g_{1}-\cos \omega_{*}\right)\right\} d \mu, \\
& v_{\theta}=\frac{A P}{a} \int_{0}^{2 \pi} \frac{1}{h}\left\{B g_{2}+\frac{1}{h^{2}} a \Lambda_{*}\left(\eta g_{1}-\cos \omega_{*}\right)\right\} d \mu .
\end{aligned}
$$

\section{Evaluation of the Integrals}

When the expressions (4.5) and (4.7) are inserted for $\Lambda_{*}, g_{1}$ and $g_{2}$, it will be seen that $v_{\tau}$ and $v_{\theta}$ consist of sums of terms of the form

$$
I_{n}^{m}(\eta, \theta, \phi, \alpha)=\int_{0}^{2 \pi} \frac{\cos ^{m}(\mu-\phi) d \mu}{\left[1+\eta^{2}-2 \eta \cos \omega_{*}\right]^{\frac{n}{2}}}, \quad m=0,1,2 ; \quad n=1,3,
$$

with coefficients which are functions of $\alpha, \beta, \theta$, and $\eta$. If we let

$$
p^{2}=h_{\max }^{2}=1+\eta^{2}-2 \eta \cos (\alpha+\theta), \quad q^{2}=h_{\min }^{2}=1+\eta^{2}-2 \eta \cos (\alpha-\theta),
$$

then

$$
h^{2}=p^{2} \sin ^{2} \frac{\mu-\phi}{2}+q^{2} \cos ^{2} \frac{\mu-\phi}{2},
$$


and it is easy to show that

$$
I_{n}^{m}=2 \int_{0}^{2 \pi} \frac{\cos ^{m} \xi d \xi}{\left[p^{2} \sin ^{2} \frac{\xi}{2}+q^{2} \cos ^{2} \frac{\xi}{2}\right]^{\frac{n}{2}}} .
$$

These integrals are evaluable in terms of complete elliptic integrals of the first and second kinds [8]; specifically,

$$
\begin{aligned}
I_{1}^{0} & =\frac{4}{p} F\left(\frac{\pi}{2}, k\right), \\
I_{3}^{0} & =\frac{4}{p^{3} k^{\prime 2}} E\left(\frac{\pi}{2}, k\right), \\
I_{1}^{1} & =\frac{4}{p}\left[\frac{1+k^{\prime 2}}{k^{2}} F\left(\frac{\pi}{2}, k\right)-\frac{2}{k^{2}} E\left(\frac{\pi}{2}, k\right)\right], \\
I_{3}^{1} & =\frac{4}{p^{3}}\left[\frac{1+k^{\prime 2}}{k^{2} k^{\prime 2}} E\left(\frac{\pi}{2}, k\right)-\frac{2}{k^{2}} F\left(\frac{\pi}{2}, k\right)\right], \\
I_{3}^{2} & =\frac{4}{p^{3}}\left[\frac{\left(1+k^{\prime 2}\right)^{2}+4 k^{\prime 2}}{k^{4} k^{\prime 2}} E\left(\frac{\pi}{2}, k\right)-4 \frac{1+k^{\prime 2}}{k^{4}} F\left(\frac{\pi}{2}, k\right)\right] .
\end{aligned}
$$

where $k=\left[\left(p^{2}-q^{2}\right) / p^{2}\right]^{1 / 2}$ is the modulus of the functions $E$ and $F$, and $k^{2}+k^{\prime 2}=1$. Using these quantities, the final form of the displacements is found to be

$$
\begin{aligned}
& \begin{aligned}
& v_{r}=\frac{4 A P}{a p}\left\{B \left(K_{1} F\right.\right.-2 \sin \theta \sin (\alpha+\beta)) \frac{E}{k^{2}} \\
&+ \frac{1}{p^{2}}\left[\frac{E}{k^{\prime 2}}\left(K_{1} \eta^{2}+\left[\cos \beta-\eta K_{1}\right] K_{2}-\frac{4 k^{\prime 2} \eta}{k^{4}} \sin ^{2} \theta \sin \alpha \sin (\alpha+\beta)\right)\right. \\
&\left.\left.\quad-\frac{2 F}{k^{2}}\left(\sin \theta\left[\sin \alpha \cos \beta+\eta^{2} \sin (\alpha+\beta)\right]-\eta \sin \theta\left[K_{1} \sin \alpha+K_{2} \sin (\alpha+\beta)\right]\right)\right]\right\}, \\
& v_{\theta}=\frac{4 A P}{a p}\left\{B\left(K_{3} F-2 \cos \theta \sin (\alpha+\beta) \frac{E}{k^{2}}\right)+\frac{1}{p^{2}}\left[\frac{E}{k^{\prime 2}}\left(\left[\cos \beta-\eta K_{1}\right] K_{4}-\frac{4 k^{\prime 2} \eta}{k^{4}} \sin \theta \cos \theta \sin (\alpha+\beta)\right)\right.\right. \\
&\left.\left.+\frac{2 F}{k^{2}}\left(-\cos \theta \sin \alpha \cos \beta+\eta\left[K_{1} \cos \theta \sin \alpha+K_{4} \sin \theta \sin (\alpha+\beta)\right]\right)\right]\right\}
\end{aligned}
\end{aligned}
$$

where we have introduced the abbreviations

$$
\begin{aligned}
& K_{1}=\cos \theta \cos (\alpha+\beta)+\frac{1+k^{\prime 2}}{2} \sin \theta \sin (\alpha+\beta), \\
& K_{2}=\cos \theta \cos \alpha+\frac{1+k^{\prime 2}}{k^{2}} \sin \theta \sin \alpha, \\
& K_{3}=-\sin \theta \cos (\alpha+\beta)+\frac{1+k^{\prime 2}}{k^{2}} \cos \theta \sin (\alpha+\beta), \\
& K_{4}=-\sin \theta \cos \alpha+\frac{1+k^{\prime 2}}{k^{2}} \cos \theta \sin \alpha .
\end{aligned}
$$

In obtaining the results (5.5) the ring has been taken in the most general position in which the force specified acting on it can be written in terms of one coordinate. If we place the ring in a more special position, viz, in the $x_{1} x_{2}$ plane, center at the origin, then (5.5) and (5.6) become somewhat simpler. Thus 


$$
\begin{gathered}
v_{r}=\frac{4 A P}{a p}\left\{B\left(K_{1}^{\prime} F-2 \sin \theta \cos \beta \frac{E}{k^{2}}\right)+\frac{1}{p^{2}}\left[\frac{E}{k^{\prime 2}}\left(K_{1}^{\prime} \eta^{2}+\left[\cos \beta-\eta K_{1}^{\prime}\right] K_{2}^{\prime}-\frac{4 k^{\prime 2} \eta}{k^{4}} \sin ^{2} \theta \cos \beta\right)\right.\right. \\
\left.\left.-\frac{2 F}{k^{2}}\left(\sin \theta \cos \beta\left[1+\eta^{2}\right]-\eta \sin \theta\left[K_{1}^{\prime}+K_{2}^{\prime} \cos \beta\right]\right)\right]\right\}, \\
v_{\theta}=\frac{4 A P}{a p}\left\{B\left(K_{3}^{\prime} F-2 \cos \theta \cos \beta \frac{E}{k^{2}}\right)+\frac{1}{p^{2}}\left[\frac{E}{k^{\prime 2}}\left(\left[\cos \beta-\eta K_{1}^{\prime}\right] K_{4}^{\prime}-\frac{4 k^{\prime 2}}{k^{4}} \eta \sin \theta \cos \theta \cos \beta\right)\right.\right. \\
\left.\left.+\frac{2 F}{k^{2}}\left(-\cos \theta \cos \beta+\eta\left[K_{1}^{\prime} \cos \theta+K_{4}^{\prime} \sin \theta \cos \beta\right]\right)\right]\right\}
\end{gathered}
$$

where

$$
\begin{aligned}
& K_{1}^{\prime}=-\cos \theta \sin \beta+\frac{1+k^{\prime 2}}{k^{2}} \sin \theta \cos \beta, \\
& K_{2}^{\prime}=\frac{1+k^{\prime 2}}{k^{2}} \sin \theta \\
& K_{3}^{\prime}=\sin \theta \sin \beta+\frac{1+k^{\prime 2}}{k^{2}} \cos \theta \cos \beta, \\
& K_{4}^{\prime}=\frac{1+k^{\prime 2}}{k^{2}} \cos \theta .
\end{aligned}
$$

\section{A Limiting Form of the Displacements}

Let the ring be located as above, and let $\beta=\pi / 2$. The force $P$ at each point of the ring is now normal to the $x_{1} x_{2}$ plane and has the sense of decreasing $x_{3}$. The distribution thus has a resultant force $2 \pi a P=\bar{P}$. At distances far removed from the ring, it may be expected that the distribution on the ring will have essentially the same effect as a concentrated force $\bar{P}$ located at the origin. We show that this is the case.

If we let the point $(r, \theta, \phi)$ recede to infinity, then $\lim _{r \rightarrow \infty} 1 / \eta=0$, and we see from (5.2) that for $r \gg a$

$$
p=\eta+\epsilon_{1}, \quad q=\eta+\epsilon_{2}
$$

where $\lim _{r \rightarrow \infty} \epsilon_{1}=\lim _{r \rightarrow \infty} \epsilon_{2}=0$. Moreover,

$$
k^{2}=\frac{p^{2}-q^{2}}{p^{2}}=\frac{4 \sin \theta}{\eta}
$$

from which we see that $\lim _{r \rightarrow \infty} k=0$, and hence that for $r \gg a$

$$
\begin{gathered}
E\left(\frac{\pi}{2}, k\right)=\frac{\pi}{2}+\epsilon_{3}, \quad F\left(\frac{\pi}{2}, k\right)=\frac{\pi}{2}+\epsilon_{4}, \\
p k^{2}=4 \sin \theta+\epsilon_{5}
\end{gathered}
$$

where $\lim _{r \rightarrow \infty} \epsilon_{i}=0, i=3,4,5$.

Using these results, it can be shown that for $r \gg a$

$$
v_{r} \simeq-A(B+1) \bar{P} \frac{\cos \theta}{r}, \quad v_{\theta} \simeq A B \bar{P} \frac{\sin \theta}{r} .
$$


If these are converted to cartesian coordinates, we find that for $r \gg a$

$$
v_{1} \simeq-A \bar{P} \frac{x_{1} x_{3}}{r^{3}}, \quad v_{2} \simeq-A \bar{P} \frac{x_{2} x_{3}}{r^{3}}, \quad v_{3} \simeq-A \bar{P}\left(B \frac{1}{r}+\frac{x^{2}}{r^{3}}\right)
$$

The right-hand members are the displacements produced in an infinite elastic medium by a concentrated force $\bar{P}$ at the origin directed along the negative $x_{3}$ axis [3].

\section{References}

[1] Sir William Thomson, Cambridge \& Dublin Mathematical Journal, 1848; Mathematical \& Physical Papers, vol. 1, p. 97.

[2] A. E. H. Love, Mathematical theory of elasticity, 4th ed. (Cambridge Press, 1927).

[3] I. S. Sokolnikoff, Mathematical theory of elasticity, 2d ed. (McGraw-Hill, 1956).

[4] J. Boussinesq, Applications des potentiel à l'étude de l'équilibre et du mouvement des solides élastiques (Paris, 1885).

[5] J. Dougall, A general method of solving the equations of elasticity, Proc. Edinburgh Mathematical Soc. 16,82 (1898).

[6] R. Mindlin, Force at a point in the interior of a semi-infinite solid, Physics, 7, 195 (1936).

[7] A. E. H. Love, A treatise on the mathematical theory of elasticity, 4th ed. (reprinted by Dover Publications, New York, N. Y., 1944).

[8] P. F. Byrd and M. D. Friedman, Handbook of Elliptic Integrals for Engineers and Physicists (Springer, Berlin, Germany, 1954).

Washington, June 19, 1957. 